\title{
A NEW PROOF OF SCATTERING BELOW THE GROUND STATE FOR THE 3D RADIAL FOCUSING CUBIC NLS
}

\author{
BENJAMIN DODSON AND JASON MURPHY \\ (Communicated by Joachim Krieger)
}

\begin{abstract}
We revisit the scattering result of Holmer and Roudenko (2008) on the radial focusing cubic NLS in three space dimensions. Using the radial Sobolev embedding and a virial/Morawetz estimate, we give a simple proof of scattering below the ground state that avoids the use of concentration compactness.
\end{abstract}

\section{INTRODUCTION}

We consider the initial-value problem for the focusing cubic nonlinear Schrödinger equation (NLS) in three space dimensions:

$$
\left\{\begin{array}{l}
\left(i \partial_{t}+\Delta\right) u=-|u|^{2} u \\
u(0)=u_{0} \in H_{x}^{1}\left(\mathbb{R}^{3}\right)
\end{array}\right.
$$

where $u: \mathbb{R}_{t} \times \mathbb{R}_{x}^{3} \rightarrow \mathbb{C}$. Solutions to (1.1) conserve the mass, defined by

$$
M(u(t)):=\int_{\mathbb{R}^{3}}|u(t, x)|^{2} d x
$$

and the energy, defined as the sum of the kinetic and potential energies:

$$
E(u(t)):=\int_{\mathbb{R}^{3}} \frac{1}{2}|\nabla u(t, x)|^{2}-\frac{1}{4}|u(t, x)|^{4} d x .
$$

We call (1.1) $\dot{H}^{1 / 2}$-critical, as the $\dot{H}^{1 / 2}$-norm of initial data is invariant under the scaling that preserves the class of solutions, namely,

$$
u(t, x) \mapsto \lambda u\left(\lambda^{2} t, \lambda x\right) .
$$

By a solution to (1.1), we mean a function $u \in C_{t} H_{x}^{1}\left(I \times \mathbb{R}^{3}\right)$ on some interval $I \ni 0$ that satisfies the Duhamel formula

$$
u(t)=e^{i t \Delta} u_{0}+i \int_{0}^{t} e^{i(t-s) \Delta}\left(|u|^{2} u\right)(s) d s
$$

for $t \in I$, where $e^{i t \Delta}$ is the Schrödinger group. If $I=\mathbb{R}$, we call $u$ global. A global solution $u$ to (1.1) scatters if there exist $u_{ \pm} \in H_{x}^{1}\left(\mathbb{R}^{3}\right)$ such that

$$
\lim _{t \rightarrow \pm \infty}\left\|u(t)-e^{i t \Delta} u_{ \pm}\right\|_{H_{x}^{1}\left(\mathbb{R}^{3}\right)}=0 .
$$

Equation (1.1) admits a global but nonscattering solution

$$
u(t, x)=e^{i t} Q(x),
$$

Received by the editors December 13, 2016.

2010 Mathematics Subject Classification. Primary 35Q55. 
where $Q$ is the ground state, i.e., the unique positive, decaying solution to the elliptic equation

$$
-\Delta Q+Q-Q^{3}=0 .
$$

Holmer and Roudenko [5] proved the following scattering result:

Theorem 1.1. Suppose $u_{0}$ is radial and $M\left(u_{0}\right) E\left(u_{0}\right)<M(Q) E(Q)$.

If $\left\|u_{0}\right\|_{L_{x}^{2}}\left\|u_{0}\right\|_{\dot{H}_{x}^{1}}<\|Q\|_{L_{x}^{2}}\|Q\|_{\dot{H}_{x}^{1}}$, then the solution to (1.1) is global and scatters.

The proof in [5] was based on the concentration compactness approach to induction on energy. In this note, we present a simplified proof of Theorem 1.1 that avoids concentration compactness. We use the radial Sobolev embedding to establish a virial/Morawetz estimate, which in turn implies 'energy evacuation' as $t \rightarrow \infty$. Together with a scattering criterion introduced by Tao [6] (see Lemma 2.2), this suffices to prove Theorem 1.1 .

Remark 1.2. In [2], Theorem 1.1] is extended to the nonradial setting, also through the concentration compactness approach. It remains to be seen if the nonradial case can be treated without concentration compactness.

Remark 1.3. The authors of [5] proved more than just scattering. Their proof gives global space-time bounds of the form

$$
\|u\|_{L_{t, x}^{5}\left(\mathbb{R} \times \mathbb{R}^{3}\right)} \leq C\left(M(Q) E(Q)-M\left(u_{0}\right) E\left(u_{0}\right)\right)
$$

for some function $C:(0, M(Q) E(Q)) \rightarrow(0, \infty)$. We use a different scattering criterion introduced by Tao [6] (Lemma 2.2). One also obtains space-time bounds; however, as the proof will show, the bounds depend on the profile of the initial data and not just the size. In this sense, we prove something weaker than the result of [5]; however, our proof is much simpler.

Remark 1.4. The results of [2,5] were generalized to other dimensions and intercritical nonlinearities by [3,4]. It should be possible to generalize our arguments as well (at least in $d \geq 3$, where the Morawetz estimates should work out).

\section{Preliminaries}

We write $A \lesssim B$ when $A$ and $B$ are nonnegative quantities such that $A \leq C B$ for some $C>0$. We indicate dependence on parameters via subscripts, e.g., $A \lesssim u B$ indicates $A \leq C B$ for some $C=C(u)>0$. We also use the big-oh notation $\mathcal{O}$. We write

$$
\|f\|_{L_{x}^{r}}=\left(\int_{\mathbb{R}^{3}}|f(x)|^{r} d x\right)^{1 / r}, \quad\|f\|_{L_{t}^{q} L_{x}^{r}\left(I \times \mathbb{R}^{3}\right)}=\|\| f(t)\left\|_{L_{x}^{r}}\right\|_{L_{t}^{q}(I)},
$$

with the usual adjustments when $q$ or $r$ is $\infty$. We write

$$
\|f\|_{\dot{H}_{x}^{1}}=\|\nabla f\|_{L_{x}^{2}}, \quad\|f\|_{H_{x}^{1}}=\|f\|_{L_{x}^{2}}+\|\nabla f\|_{L_{x}^{2}}, \quad\|f\|_{H_{x}^{1, r}}=\|f\|_{L_{x}^{r}}+\|\nabla f\|_{L_{x}^{r}} .
$$

In this note, we restrict to radial (i.e. spherically-symmetric) solutions. The following radial Sobolev embedding (which may be deduced by the fundamental theorem of calculus and Cauchy-Schwarz) plays a crucial role:

Lemma 2.1 (Radial Sobolev embedding). For radial $f \in H^{1}\left(\mathbb{R}^{3}\right)$,

$$
\||x| f\|_{L_{x}^{\infty}} \lesssim\|f\|_{H_{x}^{1}}
$$


2.1. Local theory and a scattering criterion. The local theory for (1.1) is standard. For any $u_{0} \in H^{1}$, there exists a unique maximal-lifespan solution $u$ : $I \times \mathbb{R}^{3} \rightarrow \mathbb{C}$ to (1.1). This solution belongs to $C_{t} H_{x}^{1}\left(I \times \mathbb{R}^{3}\right)$ and conserves the mass and energy. Because (1.1) is $H^{1}$-subcritical, we have an $H^{1}$-blowup criterion, namely, we can extend the solution as long as its $H^{1}$-norm stays bounded. In particular, if $u$ remains uniformly bounded in $H^{1}$ throughout its lifespan, then it is global. For a textbook treatment, we refer the reader to [1].

In [6], Tao established a scattering criterion for radial solutions to (1.1). The following appears in [6, Theorem 1.1]; for completeness, we include a sketch of the proof. We assume familiarity with Strichartz estimates.

Lemma 2.2 (Scattering criterion, [6]). Suppose $u: \mathbb{R}_{t} \times \mathbb{R}_{x}^{3} \rightarrow \mathbb{C}$ is a radial solution to (1.1) satisfying

$$
\|u\|_{L_{t}^{\infty} H_{x}^{1}\left(\mathbb{R} \times \mathbb{R}^{3}\right)} \leq E .
$$

There exist $\varepsilon=\varepsilon(E)>0$ and $R=R(E)>0$ such that if

$$
\liminf _{t \rightarrow \infty} \int_{|x| \leq R}|u(t, x)|^{2} d x \leq \varepsilon^{2},
$$

then $u$ scatters forward in time.

Sketch of the proof. Let $0<\varepsilon<1$ and $R \geq 1$ to be chosen later. The implicit constants below may depend on $E$. By Sobolev embedding, Strichartz, and monotone convergence, we may choose $T_{0}$ large enough depending on $u_{0}$ so that

$$
\left\|e^{i t \Delta} u_{0}\right\|_{L_{t}^{4} L_{x}^{6}\left(\left[T_{0}, \infty\right) \times \mathbb{R}^{3}\right)}<\varepsilon .
$$

We may further assume $T_{0}>\varepsilon^{-1}$.

By assumption, we may choose $T>T_{0}$ so that

$$
\int \chi_{R}(x)|u(T, x)|^{2} d x \leq \varepsilon^{2},
$$

where $\chi_{R}$ is a smooth cutoff to $\{|x| \leq R\}$. Using the identity

$$
\partial_{t}|u|^{2}=-2 \nabla \cdot \operatorname{Im}(\bar{u} \nabla u)
$$

which follows from (1.1), together with integration by parts and Cauchy-Schwarz, we deduce

$$
\left.\left|\partial_{t} \int \chi_{R}(x)\right| u(t, x)\right|^{2} d x \mid \lesssim \frac{1}{R}
$$

Thus, choosing $R \gg \varepsilon^{-9 / 4}$, we find

$$
\left\|\chi_{R} u\right\|_{L_{t}^{\infty} L_{x}^{2}\left(I_{1} \times \mathbb{R}^{3}\right)} \lesssim \varepsilon, \quad \text { where } \quad I_{1}=\left[T-\varepsilon^{-\frac{1}{4}}, T\right] .
$$

Using Hölder's inequality, Sobolev embedding, and radial Sobolev embedding, we deduce

$$
\|u\|_{L_{t}^{\infty} L_{x}^{3}} \lesssim\left\|\chi_{R} u\right\|_{L_{t}^{\infty} L_{x}^{2}}^{\frac{1}{2}}\|u\|_{L_{t}^{\infty} L_{x}^{6}}^{\frac{1}{2}}+\left\|\left(1-\chi_{R}\right) u\right\|_{L_{t, x}^{\infty}}^{\frac{1}{3}}\|u\|_{L_{t}^{\infty} L_{x}^{2}}^{\frac{2}{3}} \lesssim \varepsilon^{\frac{1}{2}}+R^{-\frac{1}{3}} \lesssim \varepsilon^{\frac{1}{2}},
$$

where all space-time norms are over $I_{1} \times \mathbb{R}^{3}$.

We next use the Duhamel formula to write

$$
e^{i(t-T) \Delta} u(T)=e^{i t \Delta} u_{0}+F_{1}(t)+F_{2}(t),
$$

where

$$
F_{j}(t):=i \int_{I_{j}} e^{i(t-s) \Delta}\left(|u|^{2} u\right)(s) d s \quad \text { and } \quad I_{2}=\left[0, T-\varepsilon^{-\frac{1}{4}}\right]
$$


We wish to prove

$$
\left\|e^{i(t-T) \Delta} u(T)\right\|_{L_{t}^{4} L_{x}^{6}\left([T, \infty) \times \mathbb{R}^{3}\right)} \lesssim \varepsilon^{\frac{1}{32}} .
$$

Once (2.1) is proven, one can then take $\varepsilon$ sufficiently small and use a continuity argument to deduce

$$
\|u\|_{L_{t}^{4} L_{x}^{6}\left([T, \infty) \times \mathbb{R}^{3}\right)} \lesssim \varepsilon^{\frac{1}{32}} .
$$

By standard arguments, such a bound suffices to establish scattering. Thus it remains to prove (2.1).

It is not difficult to prove by a continuity argument, Sobolev embedding, and Strichartz estimates, that

$$
\|u\|_{L_{t}^{2} L_{x}^{\infty}\left(I \times \mathbb{R}^{3}\right)}+\|u\|_{L_{t}^{2} H_{x}^{1,6}\left(I \times \mathbb{R}^{3}\right)} \lesssim(1+|I|)^{1 / 2}
$$

for any interval $I$. Thus, by Sobolev embedding and Strichartz,

$$
\left\|F_{1}\right\|_{L_{t}^{4} L_{x}^{6}\left([T, \infty) \times \mathbb{R}^{3}\right)} \lesssim \int_{I_{1}}\left\|\left(|u|^{2} u\right)(s)\right\|_{H_{x}^{1}} d s \lesssim\|u\|_{L_{t}^{\infty} L_{x}^{3}}\|u\|_{L_{t}^{2} L_{x}^{\infty}}\|u\|_{L_{t}^{2} H_{x}^{1,6}} \lesssim \varepsilon^{\frac{1}{4}},
$$

where the final three space-time norms are over $I_{1} \times \mathbb{R}^{3}$.

Next, by Hölder's inequality,

$$
\left\|F_{2}\right\|_{L_{t}^{4} L_{x}^{6}\left([T, \infty) \times \mathbb{R}^{3}\right)} \lesssim\left\|F_{2}\right\|_{L_{t}^{4} L_{x}^{3}\left([T, \infty) \times \mathbb{R}^{3}\right)}^{\frac{1}{2}}\left\|F_{2}\right\|_{L_{t}^{4} L_{x}^{\infty}\left([T, \infty) \times \mathbb{R}^{3}\right)}^{\frac{1}{2}} .
$$

Noting that

$$
F_{2}(t)=e^{i\left(t-T+\varepsilon^{-1 / 4}\right) \Delta}\left[u\left(T-\varepsilon^{-1 / 4}\right)-u_{0}\right],
$$

we can first use Strichartz to estimate

$$
\left\|F_{2}\right\|_{L_{t}^{4} L_{x}^{3}\left([T, \infty) \times \mathbb{R}^{3}\right)} \lesssim 1 .
$$

On the other hand, by the $L_{x}^{1} \rightarrow L_{x}^{\infty}$ dispersive estimate and Sobolev embedding,

$$
\left\|F_{2}(t)\right\|_{L_{x}^{\infty}} \lesssim \int_{I_{2}}|t-s|^{-\frac{3}{2}}\|u\|_{L_{t}^{\infty} H_{x}^{1}}^{3} d s \lesssim\left(t-T+\varepsilon^{-\frac{1}{4}}\right)^{-\frac{1}{2}}
$$

Thus

$$
\left\|F_{2}\right\|_{L_{t}^{4} L_{x}^{\infty}\left([T, \infty) \times \mathbb{R}^{3}\right)} \lesssim \varepsilon^{\frac{1}{16}}, \quad \text { whence } \quad\left\|F_{2}\right\|_{L_{t}^{4} L_{x}^{6}\left([T, \infty) \times \mathbb{R}^{3}\right)} \lesssim \varepsilon^{\frac{1}{32}} .
$$

Collecting the estimates yields (2.1) and thus completes the proof.

2.2. Variational analysis. We briefly review some of the variational analysis related to the ground state $Q$. For more details, see [5], 8 .

The ground state $Q$ optimizes the sharp Gagliardo-Nirenberg inequality:

$$
\|f\|_{L_{x}^{4}}^{4} \leq C_{0}\|f\|_{L_{x}^{2}}\|f\|_{\dot{H}_{x}^{1}}^{3} .
$$

The Pohozaev identities for $Q$ (which arise from multiplying (1.2) by $Q$ and $x \cdot \nabla Q$ ) imply that

$$
\|Q\|_{L_{x}^{2}}\|Q\|_{\dot{H}_{x}^{1}}=\frac{4}{3} C_{0}^{-1} \quad \text { and } \quad M(Q) E(Q)=\frac{8}{27} C_{0}^{-2} .
$$

Lemma 2.3 (Coercivity I). If $M\left(u_{0}\right) E\left(u_{0}\right)<(1-\delta) M(Q) E(Q)$ and $\left\|u_{0}\right\|_{L_{x}^{2}}\left\|u_{0}\right\|_{\dot{H}_{x}^{1}}$ $\leq\|Q\|_{L_{x}^{2}}\|Q\|_{\dot{H}_{x}^{1}}$, then there exists $\delta^{\prime}=\delta^{\prime}(\delta)>0$ so that

$$
\|u(t)\|_{L_{x}^{2}}\|u(t)\|_{\dot{H}_{x}^{1}}<\left(1-\delta^{\prime}\right)\|Q\|_{L_{x}^{2}}\|Q\|_{\dot{H}_{x}^{1}}
$$

for all $t \in I$, where $u: I \times \mathbb{R}^{3} \rightarrow \mathbb{C}$ is the maximal-lifespan solution to (1.1). In particular, $I=\mathbb{R}$ and $u$ is uniformly bounded in $H^{1}$. 
Proof. By the sharp Gagliardo-Nirenberg inequality and the conservation of mass and energy,

$$
(1-\delta) M(Q) E(Q) \geq M(u) E(u) \geq \frac{1}{2}\|u(t)\|_{L_{x}^{2}}^{2}\|u(t)\|_{\dot{H}_{x}^{1}}^{2}-\frac{1}{4} C_{0}\|u(t)\|_{L_{x}^{2}}^{3}\|u(t)\|_{\dot{H}_{x}^{1}}^{3}
$$

for $t \in I$. Using (2.2), this becomes

$$
1-\delta \geq 3\left(\frac{\|u(t)\|_{L_{x}^{2}}\|u(t)\|_{\dot{H}_{x}^{1}}}{\|Q\|_{L_{x}^{2}}\|Q\|_{\dot{H}_{x}^{1}}}\right)^{2}-2\left(\frac{\|u(t)\|_{L_{x}^{2}}\|u(t)\|_{\dot{H}_{x}^{1}}}{\|Q\|_{L_{x}^{2}}\|Q\|_{\dot{H}_{x}^{1}}}\right)^{3} .
$$

Using a continuity argument and the fact that

$$
1-\delta \geq 3 y^{2}-2 y^{3} \Longrightarrow|y-1| \geq \delta^{\prime} \quad \text { for some } \quad \delta^{\prime}>0,
$$

the first statement of the lemma follows. The second statement follows by noting that the $L^{2}$-norm is conserved and recalling the $H^{1}$ blowup criterion.

Lemma 2.4 (Coercivity II). Suppose $\|f\|_{L_{x}^{2}}\|f\|_{\dot{H}_{x}^{1}}<(1-\delta)\|Q\|_{L_{x}^{2}}\|Q\|_{\dot{H}_{x}^{1}}$. Then there exists $\delta^{\prime}=\delta^{\prime}(\delta)>0$ so that

$$
\|f\|_{\dot{H}_{x}^{1}}^{2}-\frac{3}{4}\|f\|_{L_{x}^{4}}^{4} \geq \delta^{\prime}\|f\|_{L_{x}^{4}}^{4} .
$$

Proof. Write

$$
\|f\|_{\dot{H}_{x}^{1}}^{2}-\frac{3}{4}\|f\|_{L_{x}^{4}}^{4}=3 E(f)-\frac{1}{2}\|f\|_{\dot{H}_{x}^{1}}^{2} .
$$

By the sharp Gagliardo-Nirenberg inequality and (2.2),

$$
\begin{aligned}
E(f) & \geq \frac{1}{2}\|f\|_{\dot{H}_{x}^{1}}^{2}\left[1-\frac{1}{2} C_{0}\|f\|_{L_{x}^{2}}\|f\|_{\dot{H}_{x}^{1}}\right] \\
& \geq \frac{1}{2}\|f\|_{\dot{H}_{x}^{1}}^{2}\left[1-\frac{1}{2}(1-\delta) C_{0}\|Q\|_{L_{x}^{2}}\|Q\|_{\dot{H}_{x}^{1}}\right] \geq\left(\frac{1}{6}+\frac{\delta}{3}\right)\|f\|_{\dot{H}_{x}^{1}}^{2} .
\end{aligned}
$$

Thus

$$
\|f\|_{\dot{H}_{x}^{1}}^{2}-\frac{3}{4}\|f\|_{L_{x}^{4}}^{4} \geq \delta\|f\|_{\dot{H}_{x}^{1}}^{2}, \quad \text { which implies } \quad\|f\|_{\dot{H}_{x}^{1}}^{2}-\frac{3}{4}\|f\|_{L_{x}^{4}}^{4} \geq \frac{3 \delta}{4(1-\delta)}\|f\|_{L_{x}^{4}}^{4},
$$

as desired.

\section{Proof of Theorem 1.1}

Throughout this section, we suppose $u$ to be a solution to (1.1) satisfying the hypotheses of Theorem 1.1. In particular, using the results of Section 2.2, we have that $u$ is global and uniformly bounded in $H^{1}$, and there exists $\delta>0$ such that

$$
\sup _{t \in \mathbb{R}}\|u(t)\|_{L_{x}^{2}}\|u(t)\|_{\dot{H}_{x}^{1}}<(1-2 \delta)\|Q\|_{L_{x}^{2}}\|Q\|_{\dot{H}_{x}^{1}} .
$$

We will prove that the potential energy of $u$ escapes to spatial infinity as $t \rightarrow \infty$. The same is true for the kinetic energy, but we do not need that here.

Proposition 3.1 (Energy evacuation). There exists a sequence of times $t_{n} \rightarrow \infty$ and a sequence of radii $R_{n} \rightarrow \infty$ such that

$$
\lim _{n \rightarrow \infty} \int_{|x| \leq R_{n}}\left|u\left(t_{n}, x\right)\right|^{4} d x=0 .
$$

Using Proposition 3.1, we can quickly prove Theorem 1.1. We only consider the case of scattering forward in time, as the other case is similar. 
Proof of Theorem 1.1. By Section 2.2, $u$ is global and uniformly bounded in $H^{1}$. Fix $\varepsilon$ and $R$ as in Lemma 2.2. Now take $t_{n} \rightarrow \infty$ and $R_{n} \rightarrow \infty$ as in Proposition 3.1. Then, choosing $n$ large enough that $R_{n} \geq R$, Hölder's inequality gives

$$
\int_{|x| \leq R}\left|u\left(t_{n}, x\right)\right|^{2} d x \lesssim R^{\frac{3}{2}}\left(\int_{|x| \leq R_{n}}\left|u\left(t_{n}, x\right)\right|^{4} d x\right)^{\frac{1}{2}} \rightarrow 0 \quad \text { as } \quad n \rightarrow \infty .
$$

In particular, Lemma 2.2 implies that $u$ scatters forward in time.

We prove Proposition 3.1 by a virial/Morawetz estimate. We use the virial weight in a large ball around the origin, exploiting (3.1) and coercivity to get a suitable lower bound. Spatial truncation is necessary because our solutions are merely in $H^{1}$. The large-radii terms will be treated as error terms. One needs some compactness to deduce estimates that are uniform in time. Holmer and Roudenko [5] employed concentration compactness to reduce to the study of solutions with pre-compact orbit in $H^{1}$. We will instead employ the radial Sobolev embedding and use the standard Morawetz weight at large radii.

We first need a lemma that gives (3.1) on sufficiently large balls, so that we can exhibit the necessary coercivity. We define $\chi$ to be a smooth cutoff to the set $\{|x| \leq 1\}$ and set $\chi_{R}(x):=\chi\left(\frac{x}{R}\right)$ for $R>0$.

Lemma 3.2 (Coercivity on balls). There exists $R=R(\delta, M(u), Q)>0$ sufficiently large that

$$
\sup _{t \in \mathbb{R}}\left\|\chi_{R} u(t)\right\|_{L_{x}^{2}}\left\|\chi_{R} u(t)\right\|_{\dot{H}_{x}^{1}}<(1-\delta)\|Q\|_{L_{x}^{2}}\|Q\|_{\dot{H}_{x}^{1}} .
$$

In particular, by Lemma 2.4, there exists $\delta^{\prime}=\delta^{\prime}(\delta)>0$ so that

$$
\left\|\chi_{R} u(t)\right\|_{\dot{H}_{x}^{1}}^{2}-\frac{3}{4}\left\|\chi_{R} u(t)\right\|_{L_{x}^{4}}^{4} \geq \delta^{\prime}\left\|\chi_{R} u(t)\right\|_{L_{x}^{4}}^{4}
$$

uniformly for $t \in \mathbb{R}$.

Proof. First note that

$$
\left\|\chi_{R} u(t)\right\|_{L_{x}^{2}} \leq\|u(t)\|_{L_{x}^{2}}
$$

uniformly for $t \in \mathbb{R}$. Thus, it suffices to consider the $\dot{H}^{1}$ term. For this, we will make use of the following identity, which can be checked by direct computation:

$$
\int \chi_{R}^{2}|\nabla u|^{2} d x=\int\left|\nabla\left(\chi_{R} u\right)\right|^{2}+\chi_{R} \Delta\left(\chi_{R}\right)|u|^{2} d x
$$

In particular,

$$
\left\|\chi_{R} u\right\|_{\dot{H}_{x}^{1}}^{2} \leq\|u\|_{\dot{H}_{x}^{1}}^{2}+\mathcal{O}\left(\frac{1}{R^{2}} M(u)\right) .
$$

Choosing $R$ sufficiently large depending on $\delta, M(u)$ and $Q$, the result follows.

To prove our virial/Morawetz estimate, we will use the following general identity, which follows by computing directly using (1.1).

Lemma 3.3 (Morawetz identity). Let $a: \mathbb{R}^{3} \rightarrow \mathbb{R}$ be a smooth weight. Define

$$
M(t)=2 \operatorname{Im} \int \bar{u} \nabla u \cdot \nabla a d x .
$$

Then

$$
\frac{d}{d t} M(t)=\int-|u|^{4} \Delta a+|u|^{2}(-\Delta \Delta a)+4 \operatorname{Re} a_{j k} \bar{u}_{j} u_{k} d x,
$$

where subscripts denote partial derivatives and repeated indices are summed. 
Let $R \gg 1$ to be determined below. We take $a$ to be a radial function satisfying

$$
a(x)= \begin{cases}|x|^{2} & |x| \leq \frac{R}{2} \\ 2 R|x| & |x|>R .\end{cases}
$$

In the intermediate region $\frac{R}{2}<|x| \leq R$, we impose that

$$
\partial_{r} a \geq 0, \quad \partial_{r}^{2} a \geq 0, \quad\left|\partial^{\alpha} a(x)\right| \lesssim_{\alpha} R|x|^{-|\alpha|+1} \quad \text { for } \quad|\alpha| \geq 1 .
$$

Here $\partial_{r}$ denotes the radial derivative, i.e., $\partial_{r} a=\nabla a \cdot \frac{x}{|x|}$. Under these conditions, the matrix $a_{j k}$ is nonnegative.

Note that for $|x| \leq \frac{R}{2}$, we have

$$
a_{j k}=2 \delta_{j k}, \quad \Delta a=6, \quad \Delta \Delta a=0,
$$

while for $|x|>R$, we have

$$
a_{j k}=\frac{2 R}{|x|}\left[\delta_{j k}-\frac{x_{j}}{|x|} \frac{x_{k}}{|x|}\right], \quad \Delta a=\frac{4 R}{|x|}, \quad \Delta \Delta a=0 .
$$

Proposition 3.4 (Virial/Morawetz estimate). Let $T>0$. For $R=R(\delta, M(u), Q)$ sufficiently large,

$$
\frac{1}{T} \int_{0}^{T} \int_{|x| \leq R}|u(t, x)|^{4} d x d t \lesssim u, \delta \frac{R}{T}+\frac{1}{R^{2}} .
$$

Proof. Choose $R=R(\delta, M(u), Q)$ as in Lemma3.2. We define the weight $a$ as above and define $M(t)$ as in Lemma 3.3 . Note that by Cauchy-Schwarz, the uniform $H^{1}$ bounds for $u$, and the choice of weight, we have

$$
\sup _{t \in \mathbb{R}}|M(t)| \lesssim{ }_{u} R \text {. }
$$

We compute

$$
\begin{aligned}
\frac{d}{d t} M(t)= & 8 \int_{|x| \leq \frac{R}{2}}|\nabla u|^{2}-\frac{3}{4}|u|^{4} d x \\
& +\int_{|x|>R}-\frac{4 R}{|x|}|u|^{4}+\frac{8 R}{|x|}|\not u|^{2} d x \\
& +\int_{\frac{R}{2}<|x| \leq R} 4 \operatorname{Re} a_{j k} \bar{u}_{j} u_{k}+\mathcal{O}\left(\frac{R}{|x|}|u|^{4}+\frac{R}{|x|^{3}}|u|^{2}\right) d x
\end{aligned}
$$

where $\not$ denotes the angular part of the derivative. In fact, as $u$ is radial, this term is zero. We consider (3.3) as the main term and (3.4) and (3.5) as error terms.

In (3.3) we may insert $\chi_{R}^{2}$; using (3.2) as well, we then write

$$
\left(\underline{3.3)}=8\left[\left\|\chi_{R} u\right\|_{\dot{H}_{x}^{1}}^{2}-\frac{3}{4}\left\|\chi_{R} u\right\|_{L_{x}^{4}}^{4}\right]+\int \mathcal{O}\left(\frac{1}{R^{2}}|u|^{2}\right) d x+\int \mathcal{O}\left(\chi_{R}^{4}-\chi_{R}^{2}\right)|u|^{4} d x\right. \text {. }
$$

We now apply the fundamental theorem of calculus on an interval $[0, T]$, discard positive terms, use Lemma 3.2. and rearrange. This yields

$$
\int_{0}^{T} \int \delta^{\prime}\left|\chi_{R} u(t, x)\right|^{4} d x d t \lesssim \sup _{t \in[0, T]}|M(t)|+\int_{0}^{T} \int_{|x|>R}|u(t, x)|^{4} d x+\frac{T}{R^{2}} M(u) .
$$

By the radial Sobolev embedding, we have

$$
\int_{|x|>R}|u(t, x)|^{4} d x \lesssim \frac{1}{R^{2}}\|u\|_{L_{t}^{\infty} H_{x}^{1}}^{2} M(u) .
$$


Continuing from above, we deduce

$$
\frac{1}{T} \int_{0}^{T} \int_{|x| \leq R}|u(t, x)|^{4} d x d t \lesssim u, \delta \frac{R}{T}+\frac{1}{R^{2}},
$$

as desired.

Remark 3.5. The idea of patching together the virial weight and the standard Morawetz weight is originally due to Ogawa and Tsutsumi [7]. If one worked only with a truncated virial weight, one would encounter the error term

$$
\int_{|x|>R}|\nabla u(t, x)|^{2} d x
$$

If $u$ has a pre-compact orbit in $H^{1}$, this term can be made small uniformly for $t \in \mathbb{R}$. Otherwise, it is not clear how to achieve this.

Proof of Proposition 3.1. Applying Proposition 3.4 with $T$ sufficiently large and $R=T^{1 / 3}$ implies

$$
\frac{1}{T} \int_{0}^{T} \int_{|x| \leq t^{1 / 3}}|u(t, x)|^{4} d x d t \lesssim T^{-2 / 3},
$$

which suffices to give the desired result.

\section{ACKNOWLEDGMENTS}

The first author was supported by NSF DMS-1500424. The second author was supported by the NSF Postdoctoral Fellowship DMS-1400706. This work was carried out while the authors were visiting the IMA at the University of Minnesota, which provided an ideal working environment.

\section{REFERENCES}

[1] Thierry Cazenave, Semilinear Schrödinger equations, Courant Lecture Notes in Mathematics, vol. 10, New York University, Courant Institute of Mathematical Sciences, New York; American Mathematical Society, Providence, RI, 2003. MR2002047

[2] Thomas Duyckaerts, Justin Holmer, and Svetlana Roudenko, Scattering for the non-radial $3 D$ cubic nonlinear Schrödinger equation, Math. Res. Lett. 15 (2008), no. 6, 1233-1250, DOI 10.4310/MRL.2008.v15.n6.a13. MR2470397

[3] DaoYuan Fang, Jian Xie, and Thierry Cazenave, Scattering for the focusing energy-subcritical nonlinear Schrödinger equation, Sci. China Math. 54 (2011), no. 10, 2037-2062, DOI 10.1007/s11425-011-4283-9. MR2838120

[4] Cristi Darley Guevara, Global behavior of finite energy solutions to the d-dimensional focusing nonlinear Schrödinger equation, Appl. Math. Res. Express. AMRX 2 (2014), 177-243. MR.3266698

[5] Justin Holmer and Svetlana Roudenko, A sharp condition for scattering of the radial 3D cubic nonlinear Schrödinger equation, Comm. Math. Phys. 282 (2008), no. 2, 435-467, DOI 10.1007/s00220-008-0529-y. MR.2421484

[6] Terence Tao, On the asymptotic behavior of large radial data for a focusing nonlinear Schrödinger equation, Dyn. Partial Differ. Equ. 1 (2004), no. 1, 1-48, DOI 10.4310/DPDE.2004.v1.n1.a1. MR2091393

[7] Takayoshi Ogawa and Yoshio Tsutsumi, Blow-up of $H^{1}$ solution for the nonlinear Schrödinger equation, J. Differential Equations 92 (1991), no. 2, 317-330, DOI 10.1016/00220396(91)90052-B. MR1120908

[8] Michael I. Weinstein, Nonlinear Schrödinger equations and sharp interpolation estimates, Comm. Math. Phys. 87 (1982/83), no. 4, 567-576. MR691044 
Department of Mathematics, Johns Hopkins University, John Krieger Hall, 3400 North Charles Street, Baltimore, Maryland 21218

E-mail address: bdodson4@jhu.edu

Departments of Mathematics, University of California Berkeley, 970 Evans Hall, Berkeley, California 94720

E-mail address: murphy@math.berkeley.edu 